\title{
A Probabilistic Approach for Trade-off Analysis of Composite Wing Structures at the Conceptual Phase of Design
}

\author{
Konstantinos Bacharoudis ${ }^{10000-0002-8372-9846]}$, Thomas Turner ${ }^{1[0000-0002-7997-3969]}$, Atanas \\ Popov ${ }^{1[0000-0002-3159-5647]}$, Svetan Ratchev 1[0000-0001-9955-2806] \\ ${ }^{1}$ Centre for Aerospace Manufacturing, University of Nottingham, Easter Park, Unit 4, Lenton \\ Lane, Nottingham NG7 2RD, UK \\ Konstantinos.Bacharoudis@ nottingham.ac.uk
}

\begin{abstract}
One of the major elements when performing a design for manufacturing and assembly methodology is the cost modelling method. A probabilistic cost approach is introduced herein for the series production of a composite wing structure. The proposed methodology should be able to capture changes in the design, the materials and the fabrication processes. Critically, the assembly strategy of the product should also be included to enable realistic multi-disciplinary tradeoff studies among several potential build philosophies of the wing structure at the early phase of the design. Furthermore, uncertainty related to the various input parameters, i.e. production, process and cost parameters due to incomplete knowledge, can be considered. Thus, the main effort of the present work is to set up the framework of this methodology, to develop the appropriate cost approach in order to capture manufacturing and assembly costs and further to establish a sensitivity analysis module in order to clarify the dominant cost-drivers of the product. To deal with the uncertainty, Monte Carlo simulation is implemented while Spearman's correlation coefficients are evaluated and used to perform the sensitivity study. The efficacy of the suggested methodology is demonstrated by comparing a traditional wing design against new more integrated manufacturing techniques, e.g. the co-curing process, for a simplified wing configuration.
\end{abstract}

Keywords: Cost modelling, DFMA, Composite wing, Process plan, Monte Carlo simulation.

\section{Introduction}

Concurrent engineering is increasingly the preferred approach to product development as a way of shortening the development time of the product. Its main characteristics can be summarised as working within multi-disciplinary teams and implementing in parallel from the very early stages of the product development. Design for manufacturing and assembly methods (DFMA) are essential features of the concurrent engineering approach and have developed and evolved over the past years. There are several available commercial DFMA methods [1] of qualitative and quantitative types, e.g. the Hitachi Assembly Evaluation Method [2], or the Boothroyd-Dewhurst DFMA approach 
[3], respectively. All these techniques help to develop a structured environment in which to evaluate designs in terms of manufacturability and assemblability, to explore feasible manufacturing/assembly solutions, to identify fabrication/assembly constraints of the draft designs, to estimate the cost implications when selecting a specific route, and to report to the design team areas for further improvements to the design. There are many challenges associated with these tasks, particularly when techniques are put in place for the development of aerospace components made of composite materials. A distinctive characteristic of processes for composite materials is that they are mainly additive processes while the material properties of the structure are determined as the part is manufactured. Furthermore, manufacturing and assembly processes for composite materials are relatively new compared to the processes used for the metallic materials and the processes lack in maturity, commonality and standardisation among different manufacturers. On the other hand, composite materials offer an overwhelming number of manufacturing routes to fabricate a given product making classification difficult. Considering at the same time strict aerospace requirements such as the demand for rapid one-way assembly, introduction of low cost flexible tooling and automation, quality assurance and control and tight tolerances of the aircraft components, the establishing of a robust and intelligent DFMA method is particularly challenging.

The main effort of the present work, as a first step toward the implementation of a DFMA methodology is to set up the framework of the cost methodology, that is, to identify and develop the appropriate cost approach. The aim is to capture manufacturing and assembly costs as well as to identify the cost-driver parameters at a phase in the design process when relatively little information is available. It should be stated that several cost modelling approaches exist for the cost estimation of aircraft component [4]. However, most of them lack a module that can deal with the uncertainty introduced in the input variables mainly due to the incomplete knowledge in the early stage of the design.

Thus, a process-based cost estimation method (PBCM) [5] is adopted and implemented. Recurring and non-recurring costs are estimated by placing an emphasis on the reasonable allocation of non-recurring cost over time as well as among the several operational steps of the candidate processes. Time equations are developed for every process step based on simple analytical equations correlating some basic process parameters with specific design variables. Process plans are developed for fabrication and assembly processes related to the composite components. Finally, to deal with the uncertainties of the input variables, a Monte Carlo simulation is implemented giving additionally the opportunity to perform a sensitivity analysis by evaluating the Spearman's rank correlation coefficients to identify the most important parameters or the so-called cost drivers. Having identifying cost drivers, more effort in their modelling can be devoted in order to improve the accuracy of the cost estimation. An illustrative, simplified example is performed to demonstrate the efficacy of the suggested methodology comparing a traditional wing design against new fabrication techniques that composite material offers, i.e. towards more integrated designs with fewer parts. 


\section{Cost Modelling Method}

Several cost estimation methods have been developed for the estimation of manufacturing and assembly costs for aircraft structures, among them one can identify analogous, parametric, activity and technical based methods [4]. Every method has advantages and disadvantages and should be carefully selected to fit the purpose. In the present work, a bottom-up approach is selected, i.e. a process-based cost method (or technical cost modelling approach) which is suitable for making trade-off analysis at the early stage of the design process, capturing differences in various designs and build philosophy strategies of a wing structure [5]. The method is based on the estimation of the recurring and non-recurring cost for every operational step of a manufacturing/assembly process. Material, labour (direct) and energy costs are considered as recurring costs in the present cost model. Non-recurring costs are the capital recovery of the machines/equipment/tooling/fixtures necessary for the production, maintenance costs of those machines and tools as well as the floor-space costs to accommodate the production.

The major steps to estimate the manufacturing and assembly costs are the identification of the operations related to the preferred processes, the estimation of the cycle time of each process in relation to the design, industrial and process parameters, the determination of the appropriate number of resources, e.g. number of tools, based on parameters such as the cycle time of every operation and finally the calculation of the costs using appropriate cost parameters.

\subsection{Process Based Cost Model}

In PBCM, the total manufacturing cost per component (or the total assembly cost per product) can be derived into the sum of the recurring and non-recurring costs by:

$C_{\text {Total }}=C_{\text {Material }}+C_{\text {Labour }}+C_{\text {Energy }}+C_{\text {Equipment }}+C_{\text {Tooling }}+C_{\text {Building }}$

To calculate each of these elements of per piece cost, the annual cost of each element is divided by the target annual production, $P V$. The process of interest, especially fabrication/assembly processes for carbon fibre reinforced plastic (CFRP) materials, can accommodate several operations/stages in order to be performed and be completed. Thus, the annual costs for each element are the sum of that element's costs calculated for each stage of the manufacturing/assembly process.

For the recurring costs, the unit method [6] is implemented. For the non-recurring costs, i.e. cost to buy and maintain machines/equipment, tools/fixtures, or cost to accommodate the production i.e. floor-space cost, the time value of money is considered, and the annual worth value is computed by summing the capital recovery $(\mathrm{CR})$ and the annual operating and maintenance costs (AOC) of the asset given by:

$A C_{\text {asset }}^{j}=C R_{\text {asset }}^{j}+A O C_{\text {asset }}^{j}$

where asset $=$ machines, tooling and building. The capital recovery is composed by the sum of the products of the initial investment, $P$ with the capital recovery factor $(A / P, i, n)$ 
and the salvage value, $S_{n}$ after $\mathrm{n}$ years with the sinking fund factor $(A / F, i, n)$. Usually, the annual operating and maintenance costs of the asset are expressed as a percentage of the capital recovery $\left(\alpha_{l} . C R\right)$, where $\alpha_{l}$ is in [\%].

It should be highlighted that the resources needed to achieve the annual production volume is of high importance. Thus, the number of parallel lines $N P L_{j}$ (or the number of machines) necessary to achieve the effective annual production volume $P V_{\text {effective }}^{j}$ calculated by the ratio of the required time per year to produce $P V^{j}{ }_{\text {effective }}$ parts divided by the annual uptime of the plant, its formulation is given by:

$N P L_{j}=\left\{\begin{array}{l}\frac{\tau_{j}}{U T_{j}} \text { non }- \text { dedicated } \\ \left\lceil\frac{\tau_{j}}{U T_{j}}\right\rceil \quad \text { dedicated }\end{array}\right.$

If the machine/equipment is dedicated to the fabrication of a part of interest then the value of Eq. (3) is rounded up to the next integer value. If the machine/equipment is not dedicated to a part of interest then a real value is calculated by Eq. (3). If the machine is shared among several operational steps or among the production of several parts, e.g. an autoclave to cure composite parts, then, in Eq. (3), $\tau_{j}$ becomes the sum, $\sum \tau$, from all the operations with which the machine was involved. Concerning the tooling/fixture, the number of tools necessary to run the production for a specified program life time $T_{P L}$ is given by:

$N T=\max \left(\left[\frac{P V_{\text {effective }}^{j_{P L}^{*}}}{T_{T L}}\right\rceil,\left\lceil B S_{j} . N P L_{j}\right\rceil\right)$

The first part in the max-function indicates the number of the tools that are necessary to produce the production volume for the lifetime of the project. The second term indicates the necessary number of tools to achieve the annual target production volume. The maximum between these two quantities is the necessary number of tools for the lifetime of the project. Where $P V^{J *}$ effective is the effective production volume for the stage of the process that the tool actually wears and $T_{T L}$ is the useful tooling/fixture life in [hits]. Tools are always considered to be dedicated to the part production.

\subsection{Monte Carlo Simulation and the Rank Correlation Coefficient}

A Monte Carlo (MC) simulation method is selected to deal with the uncertainty related to the input parameters. Design, industrial, process and cost parameters are considered as random variables in an attempt to express the degree of confidence for their value at the early stage of the design. MC is quite straightforward in its application. It is based on the random sampling of the vector of the input variables. Sample values for every random variable are formed and repetitive simulations performed through the developed process based on the cost model. A sample of values of the desired output parameter, e.g. the total cost, is obtained and statistically analysed further. A major step in 
implementing the MC method is the random number generators. MC analysis is implemented using VBA into Excel spreadsheets and thus the respective generators are used while several distributions for the input variables are given.

Further, sensitivity analysis is performed estimating the Spearman's rank correlation coefficient, see e.g. [7]. The Spearman's rank correlation coefficient is a non-parametric measure of a monotonic relationship between pair data. Its formulation is given by

$r_{s}=\frac{\operatorname{cov}\left(r g_{X}, r g_{Y}\right)}{\sigma_{r g_{X}} \sigma_{r g_{Y}}}$

Where $r g_{X}, r g_{Y}$ stand for the ranks of two samples $X_{i}$ and $Y_{i}, \operatorname{cov}\left(r g_{X}, r g_{Y}\right)$ is the covariance matrix of the rank variables, and $\sigma_{r g X}, \sigma_{r g Y}$ are the standard deviations of the rank variables. Thus, the closer $r_{s}$ to \pm 1 the stronger the monotonic relationship between the two variables. Herein, $X_{i}$ corresponds to the industrial, process and cost input variables, while $Y_{i}$ is the total cost (manufacturing and assembly cost).

\section{Case Study}

Advanced composite materials are relatively new materials, introducing specific advantages in the design of aircraft components and thus resulting in better performance and lighter structures. An area of particular current interest is in more highly integrated solutions, e.g. by using co-curing technique where the cycle time for the assembly can be reduced or eliminated. There may also be structural benefits (e.g. through a reduction in fastener count). Although these advantages clearly point towards the use of a DFMA approach, it is not obvious that the integrated design with the fewer parts will actually lead to a more economical solution overall. Therefore, there is a need for a trade-off study to be made investigating manufacturing and assembly costs, based at the same time on quantitative results. The probabilistic approach described in section 2 is implemented to investigate a traditional design approach against the new capabilities of integration that a composite material offers.

\subsection{Concepts}

The two sample configurations of this analysis are depicted in Fig 1 . The case study concerns a simplified wing structure of approximately $4 \mathrm{~m}$ length with $1 \mathrm{~m}$ chord line. For simplicity, which will be very often the case in the conceptual design phase, only basic parts are considered in the analysis and presented in Fig. 1.

The baseline concept comprises of two spar beams in "C" shape, one J-nose upper cover, a lower panel and a trailing edge cover.

The joining method of the parts is assumed to be mechanical fasteners, while all the laminates are considered monolithic. There are six interface areas (two on the suction side, i.e. upper spar flanges with the J-nose; and four in the pressure side of the wing, i.e. lower spar flanges with the J-nose, lower panel and trailing edge cover).

For the integrated concept, there are two spar beams in "L" shape, a J-nose upper cover with co-cured T-returns, a lower panel and the trailing edge cover. The joining 
method is assumed as structural adhesive bonding of the " $\mathrm{L}$ " spars to the T-returns with mechanical fasteners for the lower flanges of the spars to the mating parts.
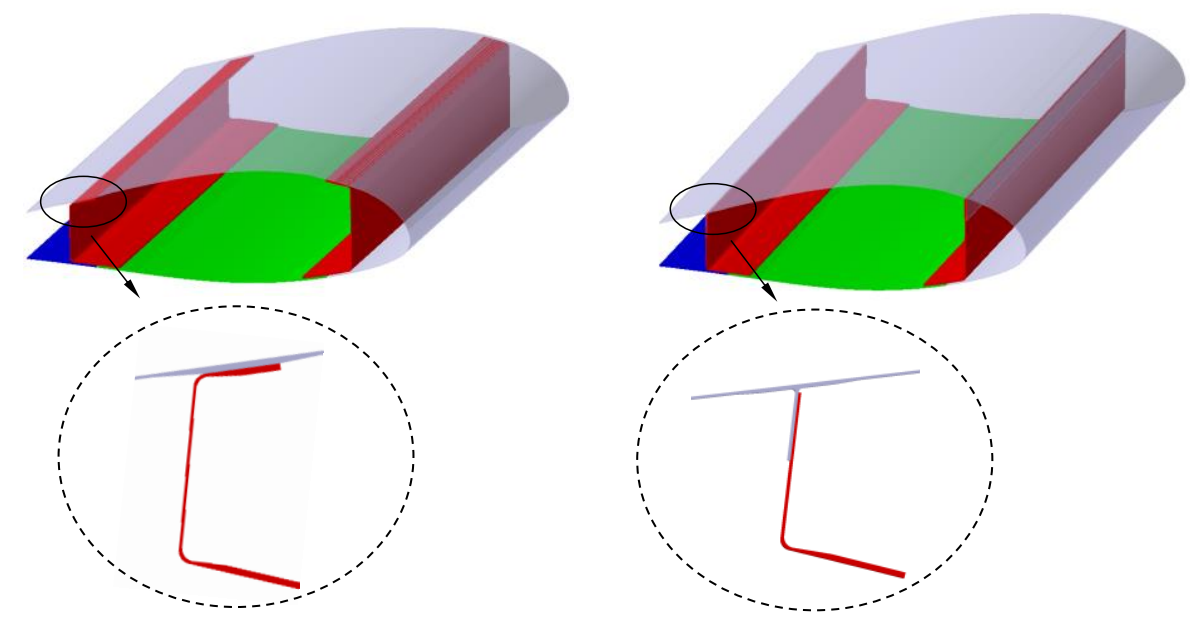

Fig. 1. Baseline (left) and Integrated (right) concept geometry

For both configurations, the most widely used process in the manufacturing of aerospace components is considered, that is, hand lay-up of carbon/epoxy prepregs on a mould, bagging and then using autoclave for curing. Additional plies for specific areas are considered for drilled mating surfaces.

It should be mentioned that co-curing is a technique where more complex, integrated components made of composite materials can be manufactured. Therefore, a more advanced tooling strategy is needed. The philosophy presented in [8] is adopted for the Jnose with the T-returns. In summary, it is assumed that five lay-up tools, one for the Jnose skin and four 'half' tools for the T-returns, and further four curing tools (caul plates) are employed. For both configuration, a manual assembly, typical for aerospace structures, is adopted.

\subsection{Scope of the Model}

The scope of the developed model is to estimate both manufacturing and assembly costs for the two concepts and further to compare and identify any benefits of using one concept over the other in terms of cost.

\subsection{Manufacturing and Assembly Process Plan}

Having determined the scope of the cost model, the first step of the methodology is to identify the necessary process steps for the fabrication of every part as well as the processes for the final assembly. Process flow diagrams are developed. They are depicted in Fig. 2 for both configurations. In this diagram, for every process step, recurring and 
non-recurring costs are indicated, while the type of the production system to be installed is also defined, e.g. cure system/metrology system, etc. It should be highlighted that the difference in the manufacturing process plan for the J-nose (baseline concept) and the co-cured J-nose is depicted by the additional red boxes in Fig. 2 . The granularity of the process plan is quite important and can be condensed as the design progresses mature and information becomes available.

Regarding the assembly process plan, similar diagrams are developed, including process steps such as shimming, drilling, deburring and sealing. The assembly diagrams accumulate almost 100 operations for the baseline configuration, while 67 operations are needed for the integrated concept.

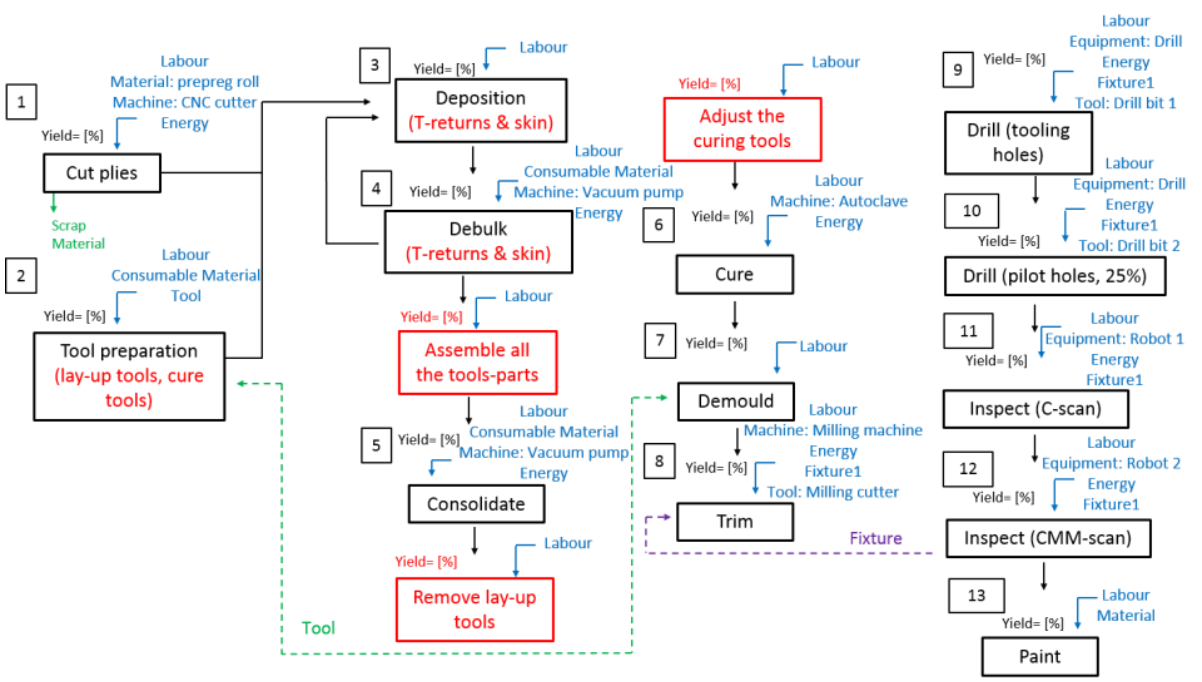

Fig. 2. Fabrication process flow for both concepts (black characters: all parts except J-nose \& Treturns, black \& red characters: J-nose \& T-returns)

The cycle time for every process step is estimated based on specific design and process parameters, e.g. part area or the lay-up rate.

Further production parameters are set up, such as the programme lifetime, the working days per year, the number of shifts per day, the paid hours per shift, the paid breaks, and the target annual production volume.

In total 66 input variables, namely industrial, process and cost parameters, are considered to be random, assuming a normal distribution with coefficient of variation ranging from $10 \%$ to $30 \%$, depending on the confidence of the modeller in their actual values. 


\section{$4 \quad$ Results and Discussion}

Results of the probabilistic analysis are presented in Fig. 3. MC was set up to perform 5,000 iterations. The mean values of the total cost of each concept (baseline or integrated) is presented with respect to the target annual production volume, the two continuous lines. Additionally, plus-minus one standard deviation of the total cost is presented in the graphs with the error bars. For clarity and to be able to visually compare the curves in Fig. 3, the annual production volume in the graph starts from 10. As expected, in this case the cost for one product per year is extremely high - almost $£ 0.4 \mathrm{M}$. Typically, aircraft production volumes go into the hundreds and thus 100 products per year is assumed as a target volume in this work.

From Fig. 3, it is clear that the most beneficial concept in terms of cost is not an easy answer and is highly dependent on the target annual production volume. More specifically, observing the mean value curves for low production volume of less than 20 products, the baseline concept offers a more attractive solution. This is due to the overwhelming manufacturing costs in the low production volumes, due to the installation of all production systems. Considering that the fabrication of the J-nose \& T-returns is more complex and thus more expensive, the baseline concept turns out cheaper for low volume production.



Fig. 3. Total cost (manufacturing and assembly costs) per product for the two concepts with respect to the target annual production volume

This trend changes when production volume is 20 to 40 products per year. Both concepts result in quite similar costs. For production volumes greater than 40 products per year, the integration concept seems to be the best approach because assembly cost is the major driver.

The total cost exhibits a rather small variation with a coefficient of variation of approximately $7 \%$, although most of the input parameters are assigned a coefficient of variation equal to $20 \%-30 \%$. 
Finally, the identification of the cost drivers is performed by estimating the rank correlation coefficients as described in Section 2. Part of the matrix is presented in Fig. 4. Input variables are listed in the first column and they are correlated with the manufacturing cost of the main components as well as the assembly cost of overall product and the total cost.

\begin{tabular}{|c|c|c|c|c|c|c|c|}
\hline RVs & $\begin{array}{c}\text { Manufacturing } \\
\text { cost (J nose) }\end{array}$ & $\begin{array}{l}\text { Manufacturing } \\
\text { cost (FW spar) }\end{array}$ & $\begin{array}{l}\text { Manufacturing } \\
\text { cost (Aft spar) }\end{array}$ & $\begin{array}{l}\text { Manufacturing } \\
\text { cost (Lower } \\
\text { cover) }\end{array}$ & $\begin{array}{l}\text { Manufacturing } \\
\text { cost (Lower } \\
\text { cover flap) }\end{array}$ & Assembly cost & Total cost \\
\hline Interest rate & 0.09 & 0.13 & 0.13 & 0.12 & 0.14 & 0.07 & 0.11 \\
\hline Labour rate - Direct & 0.78 & 0.71 & 0.67 & 0.73 & 0.66 & 0.91 & 0.89 \\
\hline Floorspace rate & -0.01 & 0.01 & 0.01 & 0.01 & 0.01 & 0.04 & 0.02 \\
\hline Electricity cost & 0.01 & 0.00 & 0.01 & 0.00 & 0.01 & 0.01 & 0.01 \\
\hline CNC cutter rate & 0.02 & 0.00 & 0.00 & 0.01 & 0.00 & 0.00 & 0.01 \\
\hline Clean tool surface rate & 0.00 & -0.01 & -0.01 & -0.01 & -0.01 & 0.00 & -0.01 \\
\hline Release agent application rate & 0.01 & -0.01 & -0.01 & 0.00 & -0.01 & 0.00 & 0.00 \\
\hline Shim application rate & 0.00 & 0.00 & 0.00 & 0.00 & -0.01 & 0.01 & 0.00 \\
\hline Hand lay-up prepreg rate & 0.11 & 0.06 & 0.20 & 0.12 & 0.19 & 0.00 & 0.08 \\
\hline
\end{tabular}

Fig. 4. Spearman's rank correlation coefficient for a subset of the random input variables for a target annual production volume equal to 100 (integrated concept)

Note that when the rank correlation coefficient value for an input variable results in a value close to unit, it means that the input variable is quite critical, highlighted with red colour in Fig. 4. Rank correlation coefficients greater than 0.2 are assumed as critical cost drivers in this exercise. The identified critical cost drivers of the integrated concept along with their rank correlation coefficient are presented in Table 1. It is obvious from Table 1 that although 66 input parameters were initially assumed, only 10 of them significantly contribute to the manufacturing/assembly costs. From Table 1 (and Fig. 4), the influence of direct labour rate is considerable and should therefore be modelled as accurately as possible. This was expected due to the high emphasis on manual activities considered both for fabrication and assembly processes. Moreover, the total cost of the integrated design concept is mainly driven just by two input parameters, namely by the labour rate and the cost of the curing system (the autoclave).

Table 1. Critical cost drivers along with their rank correlation coefficient

\begin{tabular}{lccccccc}
\hline \multicolumn{1}{c}{ Critical RVs } & $\begin{array}{c}\text { J-nose } \\
\text { Man. Cost }\end{array}$ & $\begin{array}{c}\text { FW spar } \\
\text { Man Cost }\end{array}$ & $\begin{array}{c}\text { Aft spar } \\
\text { Man Cost }\end{array}$ & $\begin{array}{c}\text { Lower } \\
\text { cover } \\
\text { Man Cost }\end{array}$ & $\begin{array}{c}\text { TE cover } \\
\text { Man Cost }\end{array}$ & $\begin{array}{c}\text { Assy } \\
\text { cost }\end{array}$ & Total \\
\hline Labour rate & 0.78 & 0.71 & 0.68 & 0.73 & 0.68 & 0.91 & 0.89 \\
HLU prepreg rate & --- & --- & 0.20 & --- & --- & --- & --- \\
CNC cutter & --- & 0.25 & 0.27 & 0.23 & 0.27 & --- & --- \\
Autoclave & --- & 0.36 & 0.4 & 0.29 & 0.40 & --- & 0.21 \\
CNC milling mach. & --- & 0.2 & 0.24 & --- & 0.24 & --- & --- \\
CNC drill & --- & 0.25 & --- & --- & 0.26 & --- & --- \\
Crane & --- & --- & --- & --- & --- & 0.28 & --- \\
Metrology system & --- & --- & --- & --- & --- & 0.20 & --- \\
AS4-12k/8552 UD & 0.26 & --- & --- & --- & --- & --- & --- \\
Assembly fixture & --- & --- & --- & --- & --- & 0.21 & --- \\
\hline
\end{tabular}

Having this information, critical input parameters can be modelled more accurately. Therefore, iterative loops of refinements can be performed as the design of the product progress, resulting in more reliable cost estimations. By assuming that a more intensive 
investigation for cost data is conducted, and by reducing the coefficient of variation of the input variables of Table 1 to their half, the statistics for every main component cost for manufacturing/assembly and overall product cost are estimated once more. Implementing this iterative loop, it was found that the associated uncertainty for the total cost is reduced almost proportionally with refinement on the critical input parameters.

\section{Conclusions}

The development of a cost tool is perhaps the most crucial element of a DFMA methodology. A process-based cost methodology has been developed to translate changes in the design, manufacturing process plan and the build philosophy of a product into cost. Furthermore, in order to deal with the uncertainty in the input parameters, which can be quite common in the conceptual phase of the design when detailed info is often missing, a Monte Carlo analysis has been implemented. Spearman's rank correlation coefficient has been evaluated to identify cost drivers and critical input parameters.

The approach has been implemented to estimate manufacturing and assembly costs of a simplified composite wing structure and to compare two different approaches, namely a baseline concept of a traditional design consisting of several parts versus integrated solutions based on co-curing techniques for composite materials. The selection of a specific concept, baseline or integrated one, is dependent on the annual production volume. The most important finding, however, of this analysis is the identification of the critical cost drivers. Having identified the critical input parameters, iterative loops of refinement can be performed as the design of the product progresses to estimate more reliable costs. The associated uncertainty is reduced almost proportionally with the refinement of the critical input parameters. It has been found that the variability on the total cost drops by half, if the variability in the critical parameters is reduced to their half.

\section{Acknowledgements}

This project has received funding from the Clean Sky 2 Joint Undertaking (JU) under grant agreement No 671436. The JU receives support from the European Union's Horizon 2020 research and innovation programme and the Clean Sky 2 JU members other than the Union.

\section{References}

1. Eskilander, S.: Design for Automatic Assembly - A method for product design: DFA2. PhD thesis, KTH Royal Institute of Technology, 2001. 
2. Miyakawa, S., Ohashi, T., Iwata, M.: The Hitachi New Assemblability Method (AEM). In: Transactions of the North American Manufacturing Research Institution of SME, pp. 352359. USA (1990)

3. Boothroyd, G., Dewhurst, P., Knight, W.: Product design for manufacture and assembly. $3^{\text {rd }}$ edition, CRC Press, (2011)

4. Hueber, C., Horejsi, K., Schledjewski, R.: Review of cost estimation: methods and models for aerospace composite manufacturing. Advanced Manufacturing: Polymer \& Composites Science. 2(1), 1-13, (2016). doi: 10.1080/20550340.2016.1154642

5. Johnson, M., Kirchain, E.: Quantifying the effects of product family decisions on material selection: A process-based costing approach. International Journal Production Economics, 120, 653-668, (2009). doi: 10.1016/j.ijpe.2009.04.014

6. Blank, L., Tarquin, A.: Engineering economy. McGraw-Hill, (2012).

7. Sheskin, D.: Handbook of parametric and non-parametric statistical procedures. $3^{\text {rd }}$ edition, CHAPMAN \& HALL/CRC, (2004)

8. Gaddiker, K., Gowda, M., Sundaram, R., Subba Rao, M.: Innovative tooling concepts for cocured composite structures in aircraft applications. International Journal of Advances in Engineering Sciences. 6(3-4), 142-147 (2014). doi: 10.1001/s12572-015-0119-0 